Pacific Journal of Mathematics

ON TWO THEOREMS OF PHRAGMÉN-LINDELÖF FOR
LINEAR ELLIPTIC AND PARABOLIC DIFFERENTIAL
EQUATIONS OF THE SECOND ORDER 


\title{
ON TWO THEOREMS OF PHRAGMÉN-LINDELÖF FOR LINEAR ELLIPTIC AND PARABOLIC DIFFERENTIAL EQUATIONS OF THE SECOND ORDER
}

\author{
AVNER Friedman
}

1. Introduction. In Part $I$ of this paper our main interest is to generalize to elliptic equations the following theorem of PhragménLindelöf :

Theorem 0. If $f(z) \rightarrow a$ as $z \rightarrow \infty$ along two straight lines, and $f(z)$ is regular and bounded in the angle between them, then $f(z) \rightarrow a$ uniformly in the whole angle as $z \rightarrow \infty$.

A generalization of the classic Phragmén-Lindelöf theorem to elliptic equations was given by Gilbarg [1] and Hopf [4]. A refined form of that classic theorem, due to the Nevanlinnas [5], [6;42-44] and Heins [3], was generalized to elliptic equations by Serrin [8].

In generalizing Theorem 0 we shall make an extensive use of the Gilbarg-Hopf results.

In Part II we generalize to parabolic equations both the classic Phragmén-Lindelöf Theorem and Theorem 0 .

In $\S 2$, Theorem 0 is proved for elliptic equations defined in any 2dimensional domains (Theorems 1,2 ). The case $n>2$ is treated in $\S 3$, for domains contained in a half space. In $\S 4$ we consider the behavior of solutions in an angular neighborhood of the origin, and we obtain results similar to those of $\S \S 2,3$. In $\S \S 5,6$, generalizations to parabolic equations are given: Theorems 7, 9 extend the classic PhragménLindelöf Theorem and Theorems 8, 10 extend Theorem 0.

The results in Part I are somewhat analogous with Theorems 2, 3, 3 of Gilbarg-Serrin's paper [2]. The similarity appears both in the type of conditions imposed on the coefficients of the elliptic operator and in the assertions. It is however important to note that our results cannot be obtained by the Gilbarg-Serrin methods, since Harnack Inequalities which play an essential role in their paper, do not hold uniformly in open domains.

Received May, 13, 1957. Paper written under contract with Office of Naval Research N58304. 


\section{PART I}

\section{Consider the differential operator}

$$
L u \equiv \sum_{i, j=1}^{n} a_{i j}(x) \frac{\partial^{2} u}{\partial x_{i} \partial x_{j}}+\sum_{i=1}^{n} b_{i}(x) \frac{\partial u}{\partial x_{i}} \quad x=\left(x_{1}, \cdots, x_{n}\right)
$$

defined in a domain $D$. In this and the following chapter $D$ is supposed to be unbounded. We denote by $\partial D$ the boundary of $D$, and by $\bar{D}$ the closure of $D$. We shall assume throughout Part I that $L$ satisfies the following conditions ([1], [4]) :

(i) $\sum_{i, j}\left|a_{i j}(x)\right|$ is bounded in $D$, and, for all $x \in D, \xi_{i}$ real,

$$
\sum_{i, j} a_{i j}(x) \xi_{i} \xi_{j} \geqq \alpha \sum_{i} \xi_{i}^{2}
$$

(ii) for all $x \in D,|x|=r$,

$$
\sum_{i}\left|b_{i}(x)\right| \leqq p(r),
$$

where $p(r)$, defined for $0<r<\infty$, is monotone decreasing and

$$
\int_{0}^{\infty} p(r) d r<\infty
$$

Define $a_{i j}(\infty)=\lim a_{i j}(x)$ as $|x| \rightarrow \infty \quad(x \in D)$, whenever the limit exists. The matrix $\left(a_{i j}(x)\right)$ is said to be Dini continuous at infinity, if there exists a monotone decreasing function $\varphi(r)$ with $\int^{\infty} r^{-1} \varphi(r) d r<\infty$, such that for $x \in D,|x|=r$,

$$
\sum_{i, j}\left|a_{i j}(x)-a_{i j}(\infty)\right| \leqq \varphi(r) .
$$

Let $u(x)$ be defined in $D$ and belong to $C^{2}(D)$. In Theorems 1-6 the function $u(x)$ is also assumed to be continuous in $\bar{D}$. Denote

$$
m(r)=\inf _{x \in D,|x|=r} u(x), \quad \mu(r)=\sup _{x \in D,|x|=r}|u(x)| .
$$

Let $K_{\beta}$ denote the $n$-dimensional cone with angular opening $\beta, 0<\beta \leqq 2 \pi$, whose axis is the positive $x_{n}$-axis and whose vertex is at the origin.

Lemma 1. Suppose $D \subset K_{\beta}, n=2$. Assume that $L$ satisfies (i), (ii) and that $\left(a_{i j}(x)\right)$ is continuous at infinity with $a_{i j}(\infty)=\delta_{i j}$. If $L u(x) \leqq 0$ in the open set $D_{r_{0}}=D \cap|x|>r_{0}, u(x) \geqq 0$ on $\partial D_{r_{0}}$ and for some $\gamma^{\prime}<\gamma=\pi / \beta$,

$$
\lim _{k \rightarrow \infty} r_{k}^{-\gamma^{\prime}} m\left(r_{k}\right)=0 \quad\left(r_{k} \rightarrow \infty \text { as } k \rightarrow \infty\right),
$$


and if $r_{0}$ is sufficiently large (depending only on $L, \beta$ and $\gamma^{\prime}$ ), then $u(x) \geqq 0$ in $D_{r_{0}}$.

By $u(x) \geqq 0$ on $\partial G$ we mean : $\lim \inf u(x) \geqq 0$ as $x$ tends to $\partial G(x \in G)$.

Proof. Following the Gilbarg-Hopf methock, it is enough to prove the existence of functions $v_{R}(x), r_{0}<R<\infty$, with the following properties:

$$
\begin{aligned}
& v_{R}(x) \geqq 0 \quad \text { if } \quad|x| \leqq R, \quad x \in \partial D_{r_{0}} \\
& v_{R}(x)=1 \quad \text { if } \quad|x|=R, \quad x \in D_{r_{0}},
\end{aligned}
$$

$$
L v_{R}(x) \leqq 0 \quad \text { if } \quad|x|<R, \quad x \in D_{r_{0}} \text {, }
$$

Denote by $h\left(x_{1}^{\prime}, x_{2}^{\prime}\right)$ the harmonic function defined in the semicircle $C^{\prime}: x_{1}^{\prime 2}+x_{2}^{\prime 2}<1, \quad x_{2}^{\prime}>0$, which takes the value 0 on the diameter and the value 1 on the rest of the boundary. The transformation $z^{\prime}=z^{\delta}$, where $\gamma^{\prime}<\delta<\gamma, z^{\prime}=x_{2}^{\prime}+i x_{1}^{\prime}, z=x_{2}+i x_{1}$, maps $S=K_{\beta} \cap|x|<1$ onto a domain $S^{\prime} \subset C^{\prime}$. The function $k\left(x_{1}, x_{2}\right)=h\left(x_{1}^{\prime}, x_{2}^{\prime}\right)$ is harmonic in $S$ and takes boundary values $\geqq 0$ on the radii and the value 1 on the rest of the boundary. We shall find $v_{R}(x)$ in the form $v_{R}(x)=f_{R}\left(k\left(\frac{x}{R}\right)\right)$.

If we show, in addition to $L f_{R} \leqq 0$, that

$$
\begin{aligned}
& f_{R}(0)=0, \quad f_{R}(1)=1, \quad 0 \leqq f_{R}(k) \leqq 1 \text { if } 0 \leqq k \leqq 1, \text { and } \\
& f_{R}(k)=0\left(k^{\gamma^{\prime} / \delta}\right) \text { uniformly in } R, \text { as } k \rightarrow 0,
\end{aligned}
$$

then (3), (4), (5) follow. Note, in proving (5), that $R^{\delta} k\left(\frac{x}{R}\right)$ is bounded as $R \rightarrow \infty$. The construction of $f_{R}$ proceeds as in Hopf's proof [4], except for the facts that property d) p. 421 and the inequality

$$
\sum_{i, j} \frac{\left|h_{i j}^{\prime \prime}(x)\right|}{\left|h^{\prime}(x)\right|^{2}}<C \quad 0<|x|<1
$$

do not hold for the corresponding $k$.

The image of $K_{\beta}$ under the mapping $z^{\prime}=z^{\delta}$ is a 2-dimensional cone $K_{\pi-\varepsilon}^{\prime}(\varepsilon>0)$ with opening $\pi-\varepsilon$ and $S^{\prime} \subset K_{\pi-\varepsilon}^{\prime}$. From Hopf's proof it is clear that instead of satisfying $d$ ), it is enough for $k$ to satisfy:

$\mathrm{d}^{\prime}$ ) along each equipotential arc $k(x)=$ const.,

$$
\left|k^{\prime}(x)\right|=\left(\sum\left(\frac{\partial k(x)}{\partial x_{i}}\right)^{2}\right)^{1 / 2} \geqq H \frac{\partial k}{\partial x_{2}}
$$

on the axis of $x_{2}$ (say at $\tilde{x}$ ), $H>0$. Since the equipotential arcs of $k(x)$ is $S$ correspond to equipotental ares of $h\left(x^{\prime}\right)$ in $S^{\prime}$, we have 


$$
\begin{aligned}
\left|k^{\prime}(x)\right|=\left|h^{\prime}\left(x^{\prime}\right)\right|\left|\frac{d z^{\prime}}{d z}\right| & \geqq \frac{\partial h\left(\tilde{x}^{\prime}\right)}{\partial x_{2}^{\prime}} \delta|x|^{\delta-1}=\frac{\partial h\left(\tilde{x}^{\prime}\right)}{\partial x_{2}^{\prime}} \delta\left|x^{\prime}\right|^{(\delta-1) / \delta} \\
& \geqq H \frac{\partial h\left(\tilde{x}^{\prime}\right)}{\partial x_{2}^{\prime}} \delta\left|\tilde{x}^{\prime}\right|^{\delta-1) / \delta}=H \frac{\partial k(\tilde{x})}{\partial x_{2}},
\end{aligned}
$$

where $\tilde{x}^{\prime}$ is the image of $\tilde{x}$ and $H>0$. Here, in the case $\delta<1$, we used the inequality $\left|x^{\prime}\right|<H_{1}\left|\tilde{x}^{\prime}\right|\left(H_{1}>0\right)$, noting that $S^{\prime} \subset K_{\pi-\varepsilon}^{\prime}$.

The estimation of $\sum a_{i j}(x) k_{i j}^{\prime \prime}(\xi)$ in $L k$ (see [4; p. 423]) has to be modified, since (8) does not hold for $k$. Defining

$$
\varepsilon_{i j}(x)=a_{i j}(x)-\delta_{i j}, \quad \varepsilon(r)=\sup _{x \in D,|x|=r} \sum\left|\varepsilon_{i j}(x)\right|,
$$

and using the harmonicity of $k$, we get

$$
\begin{aligned}
I=\left|k^{\prime}(\xi)\right|^{-2}\left|\sum a_{i j}(x) k_{i j}^{\prime \prime}(\xi)\right| & \leqq A C+\sum\left|\varepsilon_{i j}(x)\right| \frac{|\delta-1||\xi|^{\delta-2}}{\delta\left|h^{\prime}\left(\xi^{\prime}\right)\right||\xi|^{2(\delta-1)}} \\
& \leqq A C+\frac{B \varepsilon(r)}{2|\xi|^{\delta}}
\end{aligned}
$$

where $A$ and $B$ are constants, and $|\xi|<1$.

Using the inequality $2\left|\xi^{\prime}\right| \geqq h\left(\xi^{\prime}\right)([1 ; \mathrm{p} .414])$, we obtain

$$
I \leqq A C+B \varepsilon(r) k^{-1} \text {. }
$$

Define $r_{0}$ to be such that if $r>r_{0}$ then $B \varepsilon(r)<1-\gamma^{\prime} / \delta$. Then, the last inequality for $I$ shows that Hopf's method can be applied to prove that $L f_{R} \leqq 0$, provided that $f_{R}$ satisfy:

$$
\frac{f^{\prime \prime}(k)}{f^{\prime}(k)}=-A C-\frac{1-\gamma^{\prime} / \delta}{k}-\frac{P\left(x_{2}\right)}{H\left(\partial k(\tilde{x}) / \partial x_{2}\right)}, \quad f^{\prime}(k)>0,
$$

where $\tilde{x}=\left(0, x_{2}\right)$ ( $k$ is a monotone function of $\left.x_{2}\right)$.

Solving (10) we obtain,

$$
f_{R}^{\prime}(k)=E k^{\gamma^{\prime} / \delta-1} \exp \left(-A C k-P\left(x_{2}\right)\right), \quad f_{R}(0)=0,
$$

where

$$
E^{-1}=\int_{0}^{1} k^{\gamma^{\prime} / \delta-1} \exp \left(-A C-P\left(x_{2}\right)\right) d k, \quad P(s)=H^{-1} \int_{0}^{s} p(t) d t .
$$

The verification of (6), (7) is immediate and the proof is thereby completed.

Lemma 2. Suppose $D \subset K_{\beta}, n=2$. Assume that $L$ satisfies (i), (ii) and that $\left(a_{i j}(x)\right)$ is continuous at infinity with $a_{i j}(\infty)=\delta_{i j}$. If $r_{0}$ is sufficiently large, then there exists a function $w(x)$, defined in $D_{r_{0}}$, and having the following properties: 
(a) $w(x) \geqq 0$ if $\quad x \in \partial D_{r_{0}}$,

(b) $w(x)=1$ if $x \in D, \quad|x|=r_{0}$,

(c) $\quad L w(x) \leqq 0$ if $x \in D_{r_{0}}$, and

(d) $\quad w(x) \rightarrow 0$ uniformly in $D_{r_{0}}$ as $|x| \rightarrow \infty$.

Proof. To prove the lemma, define $\tilde{v}\left(x^{\prime}\right)=\frac{2}{\pi} \vartheta\left(x^{\prime}\right)$, where $\vartheta\left(x^{\prime}\right)$ is the polar angle of the point $x^{\prime}$ with $\left(-r_{0}^{\prime}, r_{0}^{\prime}\right)$ as a pole. Define also $v(x)=\tilde{v}\left(x^{\prime}\right)$, where $x^{\prime}$ is the image of $x$ under the mapping $z^{\prime}=z^{\gamma}$, where $\gamma=\pi / \beta, z^{\prime}=x_{2}^{\prime}+i x_{1}^{\prime}, z=x_{2}+i x_{1}$. We try to find $w$ in the form $w=f(v)$.

(c) implies that

$$
f^{\prime \prime}(v) \sum_{i, j} a_{i j}(x) \frac{\partial v}{\partial x_{i}} \frac{\partial v}{\partial x_{j}}+f^{\prime}(v)\left(\sum_{i, j} a_{i j}(x) \frac{\partial^{2} v}{\partial x_{i} \partial x_{j}}+\sum_{i} b_{i}(x) \frac{\partial v}{\partial x_{i}}\right) \leqq 0 .
$$

Using the harmonicity of $v(x)$ we conclude, after some calculations (see $[1 ;$ p. 414]), that (12) is a consequence of the inequalities:

$$
\frac{f^{\prime \prime}(v)}{f^{\prime}(v)}<-A_{1} \varepsilon(|x|) \frac{\left|z^{\prime}\right|}{r_{0}^{\prime}}-A_{2}|x| p(|x|) \frac{\left|z^{\prime}\right|}{r_{0}^{\prime}}, \quad f^{\prime}(v)>0,
$$

where $A_{1}, A_{2}$ are proper constants and $\varepsilon(r)$ is defined by $(g)$.

Taking $r_{0}$ to be such that $2 A_{1} \varepsilon(r)+2 A_{2} r p(r)<1-\delta(0<\delta<1)$ if $r>r_{0}$ (note that $r p(r) \rightarrow 0$ ), and using the elementary inequalities

$$
\left|z^{\prime}\right| \leqq r_{0}^{\prime} \operatorname{ctg} \vartheta^{\prime} \mid 2 \leqq 2 r_{0}^{\prime} / \widetilde{v}\left(x^{\prime}\right),
$$

we conclude that if $f(v)$ satisfies:

$$
f^{\prime \prime}(v) / f^{\prime}(v)=-(1-\delta) / v, \quad f^{\prime}(v)>0,
$$

then (13) follows. Solving (14) we find that the function $f(v)=v^{\delta}$ satisfies (a)-(d).

Theorem 1. Suppose $D \subset K_{\beta}, n=2$, and assume that $L$ satisfies (i), (ii) and that $\left(a_{i j}(x)\right)$ is continuous at infinity with $a_{i j}(\infty)=\delta_{i j}$. If $L u(x)$ $=0$ in $D$, and, for some $\eta$,

$$
\lim _{r \rightarrow \infty} \frac{\mu(r)}{r^{\pi / \beta-\eta}}=0 \quad(\eta>0 \text { if } \beta \neq \pi, \quad \eta=0 \text { if } \beta=\pi),
$$

and if $u(x) \rightarrow 0$ on $\partial D$ as $|x| \rightarrow \infty$, then $u(x) \rightarrow 0$ uniformly in $D$ as $|x| \rightarrow \infty$.

Proof. Given $\varepsilon>0$, there exists $r_{0}>0$ such that $-\varepsilon<u(x)<\varepsilon$ for 
$x \in \partial D,|x| \geqq r_{0}$. Denoting $M_{0}=\max _{|x|=r_{0}}|u(x)|$, we can apply Lemma 1 (in the case $\beta=\pi$ we apply the Gilbarg-Hopf theorem) to the function $v(x)=u(x)$ $+M_{0} w(x)+\varepsilon$ in the open set $D_{r_{0}}$. We get $v(x) \geqq 0$ in $D_{r_{0}}$. Taking $r_{1}$ to be such that $M_{0} w(x)<\varepsilon$ in $D_{r_{1}}$, we conclude that $u(x)>-2 \varepsilon$ in $D_{r_{1}}$. Similarly we get $u(x)<2 \varepsilon$ in $D_{r_{1}}$ and the theorem is proved.

REMARK. Using a proper linear transformation we conclude that the assumption $a_{i j}(\infty)=\delta_{i j}$, can be dismissed if in (15) $\beta$ is replaced by $\beta^{\prime}$, where $\beta^{\prime}$ is the angular opening of the image of $K_{\beta}$ under the linear transformation. The continuity assumption of the $a_{i j}(x)$ at infinity can be replaced by the weaker assumption that the oscillation of the $a_{i j}(x)$ near infinity is sufficiently small.

We can reduce the case $0<\beta \leqq 2 \pi$ to the case $\beta=\pi$ by the conformal mapping $z^{\prime}=z^{\pi / \beta}$, where $z=x_{2}+i x_{1}, z^{\prime}=x_{2}^{\prime}+i x_{1}^{\prime}$. Applying Theorem 1 , we get the following theorem after some calculation.

Theorem 2. Let $D \subset K_{\beta}, n=2$, and assume that $L$ satisfies (i), (ii), that $\left(a_{i j}(x)\right)$ is Dini continuous at infinity with $a_{i j}(\infty)=\delta_{i j}$, and that $r^{1-\gamma} p(r)(\gamma=\pi / \beta)$ is monotone decreasing. If $L u(x)=0$ in $D$, and

$$
\lim _{r \rightarrow \infty} \frac{\mu(r)}{r^{\pi / \beta}}=0,
$$

and if $u(x) \rightarrow 0$ on $\partial D$ as $|x| \rightarrow \infty$, then $u(x) \rightarrow 0$ uniformly in $D$ as $|x| \rightarrow \infty$.

As in Theorem 1 , the restriction $a_{i j}(\infty)=\delta_{i j}$ can be dismissed, but then in (16) and in $r^{1-\gamma} p(r), \beta$ should be replaced by $\beta^{\prime}$.

In analogue with Theorem 2, one can formulate an extension of the Gilbarg-Hopf theorem to the case $0<\beta \leqq 2 \pi$. Serrin's results [8] can also be extended to domains $D \subset K_{\beta}(0<\beta \leqq 2 \pi)$ such that the image of $D$ under the mapping $z^{\prime}=z^{\pi / \beta}$ contains a half plane $x_{2}^{\prime}>c$. In particular we have the following.

If $L u \leqq 0$ in $D$ and $u \geqq 0$ on $\partial D$, then $\lim r^{-\pi / \beta} m(r)$ exists and is $\leqq 0$.

3. In this section we consider the case $n \geqq 3$.

Lemma 3. Suppose $D \subset K_{\beta}, \frac{\pi}{3} \leqq \beta<\pi, n \geqq 3$. Assume that $L$ satisfies (i), (ii) and that $\left(a_{i j}(x)\right)$ is continuous at infinity with $a_{i j}(\infty)=\delta_{i j}$. If $L u(x) \leqq 0$ in $D_{r_{0}}, u(x) \geqq 0$ on $\partial D_{r_{0}}$, and, for some $\gamma^{\prime}<\gamma=\pi / \beta$,

$$
\lim _{k \rightarrow \infty} r_{k}^{-\gamma^{\prime}} m\left(r_{k}\right)=0 \quad\left(r_{k} \rightarrow \infty \text { as } k \rightarrow \infty\right),
$$


and if $r_{0}$ is sufficiently large, then $u(x) \geqq 0$ in $D_{r_{0}}$.

Proof. The proof proceeds as in Lemma 1, if (following Hopf [4]), we define

$$
K(x)=k\left(\rho, x_{n}\right), \quad \rho=\sqrt{x_{1}^{2}+\cdots+x_{n-1}^{2}}=\sqrt{r^{2}-x_{n}^{2}}, \quad 0<r<1,
$$

where $k$ is the function defined in the proof of Lemma 1 . The only essential difference will be in estimating $\sum a_{i j}(x) K_{i j}^{\prime \prime}(\xi)$. Clearly,

$$
\sum K_{i i}^{\prime \prime}(x)=(n-2) \frac{1}{\rho} \frac{\partial k}{\partial \rho},
$$

and

$$
\sum\left|K_{i j}^{\prime \prime}(x)\right| \leqq A_{3} \sum\left|k_{i j}^{\prime \prime}\right|+A_{4} \frac{1}{\rho}\left|\frac{\partial k}{\partial \rho}\right| \quad\left(|x|<1, A_{3}>0, A_{4}>0\right) .
$$

If we show that

$$
J \equiv \frac{1}{\rho} \frac{\partial k}{\partial \rho} /\left|k^{\prime}\right|^{2} \leqq B_{1} \quad \text { and } \quad|J| \leqq B_{1}+\frac{B_{2}}{k},
$$

where $B_{1}$ and $B_{2}$ are positive constants, then we can proceed as in the proof of Lemma 1 , and the proof of Lemma 3 will be completed.

To prove the first part of (17), we write $J$ in the form

$$
\begin{aligned}
J=\frac{|z|^{\delta-1} \sin \delta \vartheta}{\sin \vartheta} & \frac{1}{\rho^{\prime}} \frac{|z|^{\delta-1} \cos (\delta-1) \vartheta}{\left|h^{\prime}\left(z^{\prime}\right)\right|^{2} \delta|z|^{2(\delta-1)}} \frac{\partial h}{\partial \rho^{\prime}} \\
& -\frac{1}{|z| \sin \vartheta} \frac{|z|^{\delta-1} \sin (\delta-1) \vartheta}{\left|h^{\prime}\left(z^{\prime}\right)\right|^{2} \delta|z|^{2(\delta-1)}} \frac{\partial h}{\partial x_{n}^{\prime}}=J_{1}+J_{2}
\end{aligned}
$$

where $J_{1}$ is the first term and $z^{\prime}=z^{\delta}, z=x_{n}+i \rho, z^{\prime}=x_{n}^{\prime}+i \rho^{\prime}, \rho=|z| \sin \vartheta$, etc.. Since $\frac{1}{\rho^{\prime}} \frac{\partial h}{\partial \rho^{\prime}}$ is bounded near $\rho^{\prime}=0$, and since $\left|h^{\prime}\left(z^{\prime}\right)\right|$ is bounded from below by a positive constant, we get $\left|J_{1}\right| \leqq B_{1}$.

Since $\frac{\partial h\left(z^{\prime}\right)}{\partial x_{n}^{\prime}} \geqq 0$ and $\frac{\sin (\delta-1) \vartheta}{\sin \vartheta} \geqq 0$ if $1<\delta<3$ (since $1<\gamma \leqq 3$ we can take $1<\delta<3$ ), it follows that $J_{2} \leqq 0$ and consequently, $J \leqq B_{1}$.

The second part of (17) follows from noting that $\left|J_{2}\right| \leqq \frac{B_{2}}{2|z|^{\delta}} \leqq \frac{B_{2}}{k}$.

Lemma 4. Lemma 2 is true also in the case $n \geqq 3$.

Proof. The function $t(x)=r_{0}^{n-2}|x|^{2-n}$ satisfies (a), (b) and (d). We shall find $w(x)$ in the form $f(t)$. Condition (c) implies that 
(18)

$$
\begin{array}{r}
f^{\prime \prime}(t) \sum_{i, j} a_{i j}(x) \frac{(n-2)^{2} x_{i} x_{j} r_{0}^{n-2}}{|x|^{2 n}}+f^{\prime}(t)\left(\sum_{i, j} a_{i j}(x) \frac{n(n-2) x_{i} x_{j}}{|x|^{n+2}}\right. \\
\left.-\sum_{i} a_{i j}(x) \frac{n-2}{|x|^{n}}-\sum_{i} b_{i}(x) \frac{(n-2) x_{i}}{|x|^{n}}\right) \leqq 0 .
\end{array}
$$

By our assumptions, $\sum\left|a_{i j}(x)-\delta_{i j}\right| \leqq \varepsilon(|x|) \rightarrow 0$ as $|x| \rightarrow 0$. Using the harmonicity of $|x|^{2-n}$, we find that if $f(t)$ satisfies

$$
f^{\prime \prime}(t) / f^{\prime}(t)<-\left(B_{1} \varepsilon(|x|)+B_{2}|x| p(|x|)\right) / t, \quad f^{\prime}(t)>0,
$$

where $B_{1}$ and $B_{2}$ are proper constants, then (18) follows. Now, if $r_{0}$ is such that $B_{1} \varepsilon(r)+B_{2} r p(r)<1-\delta(0<\delta<1)$ for $r>r_{0}$, and if

$$
f^{\prime \prime}(t) / f^{\prime}(t)=-(1-\delta) t^{-1}, \quad f^{\prime}(t)>0,
$$

then (19) follows. Solving (20) we get the function $f(t)=t^{\delta}$, which satisfies (a)-(d).

With Lemmas 2 and 3 at hand, we can use the argument used in proving Theorem 1 and thus get the following.

Theorem 3. Suppose $D \subset K_{\beta}, \frac{\pi}{3} \leqq \beta \leqq \pi, n \geqq 3$. Assume that L satisfies (i), (ii) and that $a_{i j}(x)$ ) is continuous at infinity with $a_{i j}(\infty)=\delta_{i j}$. If $L u(x)=0$ in $D$, and for some $\eta$,

$$
\lim _{r \rightarrow \infty} \frac{\mu(r)}{r^{\pi / \beta-\eta}}=0 \quad(\eta>0 \text { if } \beta \neq \pi, \eta=0 \text { if } \beta=\pi),
$$

and if $u(x) \rightarrow 0$ on $\partial D$ as $|x| \rightarrow \infty$, then $u(x) \rightarrow 0$ uniformly in $D$ as $|x| \rightarrow \infty$.

REMARKs. (a) The remark which follows Theorem 1, applies also to Theorem 3.

(b) If we assume in Theorem 3 , that $u(x)=0\left(r^{2-n+8}\right), \delta>0$ on $\partial D$ then the same holds in $D$. This follows by applying the maximum principle to functions of the form $u(x) \pm A r^{2-n+\delta} \pm \varepsilon$, where $A$ is a proper fixed constant and $\varepsilon>0$ (compare $[2 ; 324-325]$ ).

4. Let $D$ belong to the half space $x_{n}>0$ and denote by $C_{r}$ the open set $D \cap|x|<r$. We shall consider the behavior of solutions near $x=0$; it is therefore assumed that $0 \in \bar{D}$.

We first observe that the construction of $w(x)$ in Lemma 4 , can be easily modified to derive functions $w_{r}(x)$ defined in $C_{r}^{\prime}=C_{r_{0}} \cap|x|>r$ for all $0<r<r_{0}$, and having the following properties: 
(a) $w_{r}(x) \geqq 0$ if $\quad x \in \partial C_{r}^{\prime}$,

(b) $w_{r}(x)=1$ if $x \in C_{r_{0}},|x|=r$,

(c) $L w_{r}(x) \leqq 0$ in $C_{r}^{\prime}$, and

(d) there exists $\delta(0<\delta<1)$ depending on $r_{0}\left(\delta \rightarrow 1\right.$ as $\left.r_{0} \rightarrow 0\right)$, such that

$$
\lim _{r \rightarrow 0} r^{\delta(2-n)} w_{r}(x)=0 \quad \text { if } \quad x \in C_{r_{0}} \text {; }
$$

here, $r_{0}$ is assumed to be sufficiently small, and, $\left(a_{i j}(x)\right)$ is assumed to be continuous at $x=0$ with $a_{i j}(0)=\delta_{i j}$.

With the aid of $w_{r}(x)$ we can prove an analogue of the Gilbarg-Hopf theorem.

If $L u \leqq 0$ in $C_{r_{0}}, u \geqq 0$ on $\partial C_{r_{0}}$ and

$$
\lim _{r \rightarrow 0} r^{\delta(n-2)} m(r)=0 \quad(0<\delta<1),
$$

and if $r_{0}$ is sufficiently small (depending on $\delta$ ), then $u \geqq 0$ in $C_{r_{0}}$.

We can now use the method used in proving Theorem 1, noting that the role that $w(x)$ played in that proof is now given to the function $f_{r_{0}}\left(h\left(\frac{x}{r_{0}}\right)\right)$ of Gilbarg-Hopf. The following theorem is thus proved.

Theorem 4. Let $D$ belong to the half space $x_{n}>0, n \geqq 3$. Assume that $L$ satisfies (i), (ii) and that $\left(a_{i j}(x)\right)$ is continuous at $x=0$. If $L u(x)$ $=0$ in $D$, and, for some positive $\varepsilon$,

$$
\lim _{r \rightarrow 0} r^{n-2-\varepsilon} \mu(r)=0,
$$

and if $u(x) \rightarrow 0$ on $\partial D$ as $|x| \rightarrow 0$, then $u(x) \rightarrow 0$ uniformly in $D$ as $|x| \rightarrow 0$.

The continuity assumption on the $a_{i j}(x)$ at $x=0$, can be weakened.

The case $n=2$ can be treated in a similar manner. Note that now, instead of modifying Lemma 4, we rather modify Lemma 2 and thus obtain $w_{r}(x)$ in the form $\left(\frac{2}{\pi} \vartheta\left(x_{1}^{\prime}, x_{2}^{\prime}\right)\right)^{\delta}$, where $\left(x_{1}^{\prime}, x_{2}^{\prime}\right)$ is the image of $\left(x_{1}, x_{2}\right)$ under the mapping $z^{\prime}=z^{\pi / \beta}$. We have the following.

Theorem 5. Let $D \subset K_{\beta}, n=2$, and assume that $L$ satisfies (i), (ii) and that $\left(a_{i j}(x)\right)$ is continuous at $x=0$ with $a_{i j}(0)=\delta_{i j}$. If $L u(x)=0$ in $D$, and, for some positive $\varepsilon$,

$$
\lim _{r \rightarrow 0} r^{\pi / \beta-\varepsilon} \mu(r)=0
$$


and if $u(x) \rightarrow 0$ on $\partial D$ as $|x| \rightarrow 0$, then $u(x) \rightarrow 0$ uniformly in $D$ as $|x| \rightarrow 0$.

Another way to treat the case $n=2$, is to reduce it to Theorem 1 , using the mapping $z^{\prime}=z^{-\pi / \beta}$. We thus get the following.

Theorem 6. Let $D \subset K_{\beta}, n=2$, and assume that $L$ satisfies (i), (ii) and that $\left(a_{i j}(x)\right)$ is Dini continuous at $x=0$ with $a_{i j}(0)=\delta_{i j}$. Assume further that $r^{1+\pi / \beta} p(r)$ is monotone increasing. If $L u(x)=0$ in $D$ and

$$
\lim _{r \rightarrow 0} r^{\pi / \beta} \mu(r)=0,
$$

and if $u(x) \rightarrow 0$ on $\partial D$ as $|x| \rightarrow 0$, then $u(x) \rightarrow 0$ uniformly in $D$ as $|x| \rightarrow 0$.

By using the same mapping $z^{\prime}=z^{-\pi / \beta}$, we can derive theorems analogous with the Gilbarg-Hopf ([1], [4]) and Serrin's ([8]) theorems, provided that $L$ satisfies the assumptions of Theorem 6 .

In the case $n \geqq 3, \beta \leqq \pi$, such theorems can also be obtained, by using the transformation $x_{i}^{\prime}=x_{i} /|x|^{n}(i=1, \cdots, n)$.

\section{PART II}

5. Let $x=\left(x_{1}, \cdots, x_{n}\right)$ and denote $X=(x, t),|X|=\left(|x|^{2}+t^{2}\right)^{1 / 2}$. Consider the operator

$$
L u \equiv \sum_{i, j=1}^{n} a_{i j}(X) \frac{\partial^{2} u}{\partial x_{i} \partial x_{j}}+\sum_{i=1}^{n} b_{i}(X) \frac{\partial u}{\partial x_{i}}-\frac{\partial u}{\partial t},
$$

defined in an unbounded domain $D$. We shall assume that $L$ satisfies the following conditions :

(i) $\sum_{i, j}\left|a_{i j}(X)\right|$ is bounded in $D$, and, for all $X \in D$, $\dot{\xi}_{i}$ real,

$$
\sum_{i, j} a_{i j}(X) \xi_{i} \xi_{j} \geqq \alpha \sum_{i} \xi_{i}^{2}
$$

(ii) for all $X \in D,|X|=R$,

$$
\left|\sum_{i} x_{i} b_{i}(X)\right| \leqq p(R)
$$

where $p(R)(0<R<\infty)$ is bounded and $p(R) \rightarrow 0$ as $R \rightarrow \infty$.

Beside the functions $m(R), \mu(R)$ defined in Part I, we introduce the functions

$$
m^{\prime}(R)=\inf _{x \in T_{R}} u(X), \quad \mu^{\prime}(R)=\sup _{x \in T_{R}}|u(X)|,
$$

where $T_{R} \equiv D \cap|x|^{2}+|t|=R$.

Let $K_{\beta}$ denote the cone with angular opening $\beta$, whose axis is the 
positive $t$-axis and whose vertex is in the origin. In what follows, $u(X)$ is assumed to belong to $C^{2}(D)$. In Theorems $8,10 u(X)$ is also assumed to be continuous in $\bar{D}$.

THEOREM 7. Let $D$ belong to the half space $t>0$, and assume that $L$ satisfies (i), (ii). If $u(X) \geqq 0$ on $\partial D, L u(X) \leqq 0$ in $D$, and if

$$
\lim _{k \rightarrow \infty} \frac{m\left(R_{k}\right)}{R_{k}^{2}}=0 \quad\left(R_{k} \rightarrow \infty \text { as } k \rightarrow \infty\right),
$$

then $u(X) \geqq 0$ in $D$.

Proof. The function $v_{R}(X)=\left(|x|^{2}+(t+K)^{2}\right) / R^{2}(K>0)$ has the following properties :

(a) $v_{R}(X) \geqq 0$ if $X \in \partial D,|X| \leqq R$,

(b) $v_{R}(X) \geqq 1$ if $X \in D,|X|=R$,

(c) $L v_{R}(X)<0$ in $C_{R}=D \cap|X|<R$, if $K$ is sufficiently large, and

(d) $R^{2} v_{R}(X)$ is bounded, for every $X$, as $R \rightarrow \infty$.

The function $\tilde{u}(X)=u(X)-\sigma(R) v_{R}(X)$, where $\sigma(R)=\min (0, m(R))$, is nonnegative on $\partial C_{R}$ and $L u(X) \leqq 0$ in $C_{R}$. Applying the (weak) minimum principle [7], we conclude that $\tilde{u}(X) \geqq 0$ in $C_{R}$. Taking $R=R_{k} \rightarrow \infty$ and using (3), we get $u(X) \geqq 0$.

REMARK. It is clear that the same proof holds under weaker assumptions on $L$ : (ii) may be replaced by $\sum x_{i} b_{i}(X) \leqq H$, where $H$ is a constant, and in (i), the boundedness of $\sum\left|a_{i j}(X)\right|$ in $D$ may be replaced by the boundedness of $\sum a_{i i}(X)$ in $D$ and the boundedness of $\sum\left|a_{i j}(X)\right|$ in each $C_{R}$.

Lemma 5. Let $D$ belong to the half space $t>0$, and assume that $L$ satisfies (i), (ii). If $R_{0}$ is sufficiently large, then there exists a function $w(X)$ defined in $D_{R_{0}}=D \cap|X|>R_{0}$, and having the following properties:

(a) $w(X) \geqq 0$ if $X \in \partial D_{R_{0}}$,

(b) $w(X) \geqq 1$ if $X \in D,|X|=R_{0}$,

(c) $L w(X) \leqq 0$ in $D_{R_{0}}$, and

(d) $w(X) \rightarrow 0$ uniformly in $D_{R_{0}}$ as $|X| \rightarrow \infty$. 


$$
w(X)=\frac{C}{(t+1)^{\varepsilon}} \exp \left(\frac{-H|x|^{2}}{t+1}\right) \quad(C>0, \varepsilon>0, H>0) .
$$

Since $W(X)>0$ if $|X|=R_{0}, t \geqq 0$, we can choose $C$ such that (b) is satisfied. Since (a) and (d) are also satisfied, it remains to verify (c).

$$
L w=w\left\{\sum a_{i j} \frac{4 H^{2} x_{i} x_{j}}{(t+1)^{2}}-\sum a_{i i} \frac{2 H}{t+1}-\sum x_{i} b_{i} \frac{2 H}{t+1}+\frac{\varepsilon}{t+1}-\frac{H|x|^{2}}{(t+1)^{2}}\right\} ;
$$

consequently, if

$$
4 H \sum a_{i j} x_{i} x_{j} \leqq|x|^{2}, \quad 2 H \sum a_{i i}+2 H \sum x_{i} b_{i} \geqq \varepsilon,
$$

then $L w \leqq 0$. Obviously we can choose $H$ and $\varepsilon$ such that (4) is satisfied.

With Theorem 7 and Lemma 5 at hand, we can now proceed as in the proof of Theorem 1 and get the following.

THEOREM 8. Let $D$ belong to the half space $t>0$, and assume that $L$ satisfies (i), (ii). If $L u(X)=0$ in $D$ and

$$
\lim _{R \rightarrow \infty} \frac{\mu(R)}{R^{2}}=0,
$$

and if $u(X) \rightarrow 0$ on $\partial D$ as $|X| \rightarrow \infty$, then $u(X) \rightarrow 0$ uniformly in $D$ as $|X| \rightarrow \infty$.

Theorems 7, 8 are not true for domains $D$ in the half space $t<0$. As an example take $D$ to be the whole half space $t<0$, and take $u(x, t)$ $=t^{1 / m}$, where $m$ is an odd positive integer. Then

$$
\begin{gathered}
u=0 \text { on } t=0, \quad L u=-\frac{1}{m} t^{1 / m-1}<0 \quad \text { if } t<0, \\
\lim _{R \rightarrow \infty} \frac{\mu(R)}{R^{\varepsilon}}=0 \quad \text { if } \quad \frac{1}{m}<\varepsilon,
\end{gathered}
$$

but $u(X)<0$ if $t<0$, and $\lim u(X)$ does not exist as $|X| \rightarrow \infty, t \leqq 0$.

6. ThEOREM 9. Let $D \subset K_{\beta}, 0<\beta<2 \pi$, and assume that $L$ satisfies (i), (ii). If $L u(X) \leqq 0$ in $D, u(X) \geqq 0$ on $\partial D$, and if

$$
\lim _{k \rightarrow \infty} \frac{m^{\prime}\left(R_{k}\right)}{R_{k}^{2}}=0 \quad\left(R_{k} \rightarrow \infty \text { as } k \rightarrow \infty\right),
$$

then $u(X) \geqq 0$ in $D$. 
Taking $v_{R}(X)=2\left(|x|^{2}+B t+C\right) / R^{2}$ ( $B$ and $C$ are proper constants), we proceed as in the proof of Theorem 7. Details will be omitted. The remark that follows Theorem 7 applies also to Theorem 9.

Lemma 5 can also be generalized to the case $D \subset K_{\beta}, 0<\beta<2 \pi$. Indeed, the function $w(X)$ may be defined as follows:

$$
w(X)=\left\{\begin{array}{cl}
\frac{C}{\left(t+R_{0}\right)^{\varepsilon}} \exp \left(-\frac{H|x|^{2}}{t+R_{0}}\right) & \text { if } t>-R_{0} \\
0 \quad \text { if } t \leqq-R_{0} .
\end{array}\right.
$$

Proceeding as in $\S 5$, we get the following theorem.

Theorem 10. Let $D \subset K_{\beta}, 0<\beta<2 \pi$, and assume that $L$ satisfies (i), (ii). If $L u(X)=0$ in $D$ and

$$
\lim _{R \rightarrow \infty} \frac{\mu^{\prime}(R)}{R^{2}}=0,
$$

and if $u(X) \rightarrow 0$ on $\partial D$ as $|X| \rightarrow \infty$, then $u(X) \rightarrow 0$ uniformly in $D$ as $|X| \rightarrow \infty$.

Note that (7) can be replaced by the stronger assumption

$$
\lim _{R \rightarrow \infty} \frac{\mu(R)}{R}=0 \text {. }
$$

\section{REFERENCES}

1. D. Gilbarg, The Phragmén-Lindelöf theorem for elliptic partial differential equations,

J. Rational Mech. Anal, 1 (1952), 411-417.

2. - - - and J. Serrin, On isolated singularities of solutions of second order elliptic differential equations, J. Analyse Math. 4 (1954-56), 309-340.

3. M. Heins, On the Phragmén-Lindelöf principle, Trans. Amer. Math. Soc., 60 (1946), 238-244.

4. E. Hopf, Remarks on the preceding paper by D. Gilbarg, J. Rational Mech. Anal., 1 (1952), 418-424.

5. F. \& R. Nevanlinna, Über die Eigenschaften einer analytischen Funtetion in der Umgebung einer singulären Stelle oder Linie, Acta Soc. Sci. Fenn., 50, Nr. 5 (1922).

6. R. Nevanlinna, Eindeutige analytische Funktionen, Springer, Berlin (1936).

7. L. Nirenberg, A strong maximum principle for parabolic equations, Comm. Pure Appl. Math., 6 (1953), 167-177.

8. J. B. Serrin, On the Phragmén-Lindelöf principle for elliptic differential equations,

J. Rational Mech. Anal., 3 (1954), 395-413. 



\title{
PACIFIC JOURNAL OF MATHEMATICS
}

\section{EDITORS}

H. L. RoYden

Stanford University

Stanford, California

\section{R. A. Beaumont}

University of Washington

Seattle 5 , Washington
A. L. Whiteman

University of Southern California

Los Angeles 7, California

E. G. Straus

University of California

Los Angeles 24, California

\section{ASSOCIATE EDITORS}

\author{
E. F. BECKENBACH \\ C. E. BURGESS \\ M. HALL \\ E. HEWITT
}
A. HORN
V. GANAPATHY IYER
R. D. JAMES
M. S. KNEBELMAN

L. NACHBIN

I. NIVEN

T. G. OSTROM

M. M. SCHIFFER
G. SZEKERES

F. WOLF

K. YOSIDA

\section{SUPPORTING INSTITUTIONS}

\author{
UNIVERSITY OF BRITISH COLUMBIA \\ CALIFORNIA INSTITUTE OF TECHNOLOGY \\ UNIVERSITY OF CALIFORNIA \\ MONTANA STATE UNIVERSITY \\ UNIVERSITY OF NEVADA \\ OREGON STATE COLLEGE \\ UNIVERSITY OF OREGON \\ UNIVERSITY OF SOUTHERN CALIFORNIA
}

Mathematical papers intended for publication in the Pacific Journal of Mathematics should be typewritten (double spaced), and the author should keep a complete copy. Manuscripts may be sent to any of the editors. All other communications to the editors should be addressed to the managing editor, E. G. Straus at the University of California, Los Angeles 24, California.

50 reprints per author of each article are furnished free of charge; additional copies may be obtained at cost in multiples of 50 .

The Pacific Journal of Mathematics is published quarterly, in March, June, September, and December. The price per volume (4 numbers) is $\$ 12.00$; single issues, $\$ 3.50$. Back numbers are available. Special price to individual faculty members of supporting institutions and to individual members of the American Mathematical Society: $\$ 4.00$ per volume; single issues, $\$ 1.25$.

Subscriptions, orders for back numbers, and changes of address should be sent to Pacific Journal of Mathematics, 2120 Oxford Street, Berkeley 4, California.

Printed at Kokusai Bunken Insatsusha (International Academic Printing Co., Ltd.), No. 10, 1-chome, Fujimi-cho, Chiyoda-ku, Tokyo, Japan.

PUBLISHED BY PACIFIC JOURNAL OF MATHEMATICS, A NON-PROFIT CORPORATION

The Supporting Institutions listed above contribute to the cost of publication of this Journal, but they are not owners or publishers and have no responsibility for its content or policies. 


\section{Pacific Journal of Mathematics}

\section{Vol. 7, No. 4 \\ April, 1957}

Robert Geroge Buschman, A substitution theorem for the Laplace

transformation and its generalization to transformations with

symmetric kernel .

S. D. Conte, Numerical solution of vibration problems in two space variables ...................................... 1535

Paul Dedecker, A property of differential forms in the calculus of variations ......................................... 1545

H. Delange and Heini Halberstam, A note on additive functions . . . . . . . 1551

Jerald L. Ericksen, Characteristic direction for equations of motion of non-Newtonian fluids ................................. 1557

Avner Friedman, On two theorems of Phragmén-Lindelöf for linear elliptic and parabolic differential equations of the second order ........... 1563

Ronald Kay Getoor, Additive functionals of a Markov process . . . . . . . . . 1577

U. C. Guha, $(\gamma, k)$-summability of series . . . . . . . . . . . . . . . . . . 1593

Alvin Hausner, The tauberian theorem for group algebras of vector-valued functions ...................................... 1603

Lester J. Heider, $T$-sets and abstract $(\mathrm{L})$-spaces ................. 1611

Melvin Henriksen, Some remarks on a paper of Aronszajn and

Panitchpakdi........................................ 1619

H. M. Lieberstein, On the generalized radiation problem of A. Weinstein ... 1623

Robert Osserman, On the inequality $\Delta u \geq f(u) \ldots \ldots \ldots \ldots \ldots \ldots \ldots . \ldots 1641$

Calvin R. Putnam, On semi-normal operators . . . . . . . . . . . . . . . . . . 1649

Binyamin Schwarz, Bounds for the principal frequency of the

non-homogeneous membrane and for the generalized Dirichlet integral...

Edward Silverman, Morrey's representation theorem for surfaces in metric

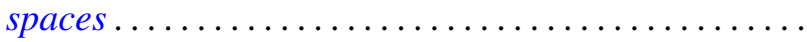

V. N. Singh, Certain generalized hypergeometric identities of the Rogers-Ramanujan type. II .

R. J. Smith, A determinant in continuous rings 1701

Drury William Wall, Sub-quasigroups of finite quasigroup 1711

Sadayuki Yamamuro, Monotone completeness of normed semi-ordered linear spaces.

C. T. Rajagopal, Simplified proofs of "Some Tauberian theorems" of Jakimovski: Addendum and corrigendum ............

N. Aronszajn and Prom Panitchpakdi, Correction to: "Extension of uniformly continuous transformations in hyperconvex metric spaces"...................................

Alfred Huber, Correction to: "The reflection principle for polyharmonic functions" 